\title{
Towards digital twins: machine learning based process coupling and multiscale modelling of reactive transport phenomena
}

\author{
NIKOLAOS I. PRASIANAKIS ${ }^{1}$
}

${ }^{1}$ Laboratory for Waste Management, Paul Scherrer Institut, CH-5232 Villigen PSI, Switzerland (nikolaos.prasianakis@psi.ch)

The recent advancements in the area of machine learning have the potential to revolutionize the traditional way of performing reactive transport simulations, for a wide range of geochemical applications, as well as the way of conducting laboratory experiments. Typical systems of interest are related to geothermal energy and oil reservoirs as well as radioactive waste geological repositories. The evolution of such systems is driven by the interplay of thermal, chemical, mechanical and hydraulic gradients. Consequently, the modelling and simulation of such increased complexity systems requires the description of several physical and chemical processes, which occur in many different spatial and temporal scales, in a coupled self-consistent approach.

Although at each specific scale e.g. the pore scale [1], the models and the experimental research tools are well established, an automated coupling between processes at different scales remains very challenging [2]. For example, the geochemical evolution of the rock microstructure can result in different permeability-porosity pathways depending on several parameters relevant to the chemical and flow conditions [3]. Parametric microscopic simulation results have to be subsequently up-scaled to be used by field-scale simulators. In this context, deep neural networks can be used as machine-native interfaces between heterogeneous codes for linking nano- to micro- and macro- scales.

At the same time, modern high performance parallel computing facilities have the capacity to compute the evolution of geochemical systems, which can be described by more than $10^{10}$ discrete computational volumes. Accelerating the geochemical computations using sufficiently trained neural networks is a key enabling step. If successful, the everincreasing realism of such simulations can lead to reliable digital twins, the necessary companion for future laboratory experimental investigations and modelling diagnostics [4].

[1] Molins, S., Soulaine, C., Prasianakis, N.I. et al. Comput. Geosci. (2020). https://doi.org/10.1007/s10596-019-09903-x [2] Churakov, S.V. \& Prasianakis, N.I. (2018) Am. J. Science 318, 921-948 [3] Prasianakis, N.I. et al. (2018) Geofluids 9260603 [4] Poonoosamy et al. (2019) Chem. Geology 528, 119264 